\title{
Are teeth in Scotland extracted for the same reasons now as 15 years ago?
}

\author{
The reasons for extraction of permanent teeth in Scotland: a 15-year follow-up study by L. K. McCaul, W. M. M. \\ Jenkins and E. J. Kay Br Dent J 2001; 190: 658-662
}

\begin{abstract}
Aims
Although Scotland has the highest proportion of edentulous adults in the UK, the frequency of edentulousness has fallen by $21 \%$ during the last 20 years. This study, carried out in 1999, was designed to establish whether the reasons for tooth loss have also changed since 1984 when they were last determined.
\end{abstract}

\author{
Methods \\ The Scottish Dental Practice Board provided the names of every \\ fourth dentist on its list among which 425 general dental \\ practitioners were identified. They were asked to record \\ permanent tooth extractions for 1 week, specifying the age, sex \\ and dental attendance of patients who underwent extractions \\ and the reasons for these extractions. 352 dentists took part: a \\ response rate of $82.8 \%$.

\section{Results} \\ The study confirmed that there has been a reduction in the \\ number of extractions between 1984 and 1999: there were 25\% \\ fewer teeth extracted per patient and 30\% fewer per dentist per \\ week. From 0-20 years of age, orthodontics has replaced caries as \\ the commonest reason for extraction and in all age groups over \\ 20 years, caries has become the commonest reason in contrast to \\ 1984 when periodontal disease was the principal reason in \\ patients over 40 years old.
}

\author{
Conclusions \\ Caries and its sequelae remain the most important cause of tooth \\ loss throughout adult life in Scotland and, therefore, caries \\ prevention and maintenance of restorations are of great \\ importance at all ages.
}

\section{Comment}

Ttraction of teeth in the UK is someEthing that, until recently, was taken for granted in dentistry, so much so that for many years no-one seemed to ask why. To try and answer the question in 1984 Kay and Blinkhorn carried out a study in Scotland to try and find out the main reasons. Not surprisingly, dental caries was found to be the main culprit, although the emphasis shifted to periodontal disease in patients aged over 40 .

This pattern led to a general perception as to which health strategies were appropriate to try and reduce tooth loss through both caries and periodontal disease, and helped mould the professional consciousness as to why dentists removed teeth from their patients.

Nearly twenty years later we knew from the Adult Dental Health Survey that the number of teeth extracted (in Scotland) had fallen from $39 \%$ in 1978 to $18 \%$ in
1998, a substantial decrease. This doubtless helped raise the question investigated in this study as to whether permanent teeth were still being extracted for the same reasons, and in the same proportions. After all, some of the health strategies put in place over the intervening years since the initial study might have worked.

This paper compares the findings with the initial study in 1984. The study confirmed the findings of The Adult Dental Health Survey by also showing a decrease in the number of teeth extracted per patient. It also demonstrated two important shifts in the reason for extraction, as removal for orthodontic treatment had replaced caries as the main reason for removing teeth in patients under 20 years, while caries had replaced periodontal disease as the main reason for removing teeth in patients over 40.

The authors speculate on these observations, commenting on how the fact that caries appears more important in oral disease in older adults compared with fifteen years ago and offering some possible reasons for this. These include the difference in patient perceptions over decision-making, improvements in periodontal diagnosis, changing dentist attitudes to periodontally-involved but symptomless teeth and economic factors.

One aspect of this study is the high response rate $(83 \%)$ which the authors attribute to good questionnaire design and followup. One point to note on this is that the protocol was slightly different in the collection of data between the 1984 study and the current one, possibly resulting in bias, and the authors sensibly urge caution in the interpretation of their results.

\section{Mike Grace}

Editor, British Dental Journal 